\title{
El usuario y su uso de las urgencias
}

\section{The user and his use of emergency services and departments}

\section{O. Miró}

Mucho han cambiado las cosas desde que aquel buen samaritano socorriese al judío apaleado que encontró en su camino, le vendara las heridas, lo subiera en su burro y lo llevara a una posada cercana donde poder atenderlo mejor ${ }^{1}$. Entre aquel sencillo acto, que muchos consideran el relato de la primera prestación de ayuda extrahospitalaria urgente recogida en la literatura, y la compleja respuesta que a día de hoy se da a la necesidad de asistencia sanitaria urgente, media un abismo. Sólo por poner un poco en perspectiva la magnitud actual de la situación: los servicios de urgencias hospitalarios (SUH) españoles atendieron más de 24 millones de consultas en 2005, lo que supone que uno de cada dos ciudadanos fueron atendidos en ellos ${ }^{2}$. Y eso sin perder de vista que las consultas urgentes realizadas en otros niveles, ya sea por los servicios de emergencias médicas o en los diferentes dispositivos dependientes de las áreas básicas de salud, superan de largo las que se realizan en los $\mathrm{SUH}^{2}$.

El equilibrio entre demanda y oferta de asistencia sanitaria urgente es difícil en todos los países y sistemas y, desde hace años, la balanza se inclina del lado de la primera ${ }^{3}$. Me centraré exclusivamente en aproximar un poco la situación en España, donde el colapso asistencial se ha convertido en una de las señas de identidad de sus $\mathrm{SUH}^{4,5}$. Aunque esto es algo que resulta ampliamente conocido, e incluso en ciertos círculos implícitamente tolerado, ya no lo son tanto las causas que conducen a este desequilibrio. Hay datos procedentes de múltiples estudios que apuntan a una excesiva demanda de atención urgente. Por ejemplo, es bien sabido que la demanda de atención urgente es en muchos casos (un $31 \%$ según en el estudio de Aranaz y col ${ }^{6}$ ) inadecua$\mathrm{da}^{7} \mathrm{o}$, incluso cuando es adecuada, el usuario hace un uso de ella en función de otras variables circunstanciales en las que en ocasiones la

Sección de Urgencias Medicina. Área de Urgencias. Hospital Clínic. Barcelona.

El Dr. Òscar Miró disfruta de una beca de Intensificación Investigadora del Instituto Carlos III durante el año 2009.

\section{Correspondencia: \\ Òscar Miró \\ Hospital Clínic \\ Área de Urgencias \\ Villarroel, 170 \\ 08036 Barcelona}

Tfno. 932279833

Fax 932275693

E-mail: omiro@clinic.ub.es 
salud no se coloca en un primer plano ${ }^{8,9}$. Otros estudios y opiniones, en cambio, han abogado por una oferta de servicios insuficiente. La población española creció un 8,9\% de 2000 a 2005 (de 40,5 a 44,1 millones de habitantes $)^{10}$. El número de consultas atendidas durante ese mismo quinquenio en los hospitales, públicos y privados, españoles creció un $10,4 \%$ (de 20,3 a 24,4 millones de consultas). Sin embargo, es obvio que los recursos para hacer frente a esta situación (mayor población con incluso mayor requerimiento de atención urgente) no han crecido a un ritmo comparable. Finalmente, por si no fuera suficiente este aumento cuantitativo de población y demanda, la población ha envejecido y muchos pacientes pluripatológicos o con enfermedades crónicas graves sobreviven ahora gracias a tratamientos más agresivos, pero a costa de un incremento en la complejidad de sus cuidados médicos en general, de sus consultas urgentes en particular, y de la necesidad de ingreso tras esa consulta urgente ${ }^{11}$.

Cualesquiera que sean las causas que subyacen, el resultado para el usuario es palpable: retrasos generalizados en recibir asistencia médica urgente, los cuales van unidos a un deterioro de la calidad en el servicio prestado ${ }^{12}$. Ha habido intentos o propuestas de incidir en ambos platos de la balanza. Por el lado de la demanda, se ha sugerido promover el copago del servicio en determinadas circunstancias, especialmente el mal uso o abuso injustificado ${ }^{13}$, redirigir las consultas inadecuadas a otros niveles asistenciales ${ }^{14} \mathrm{u}$ ofrecer servicios telefónicos o telemáticos al ciudadano que solucionen su problema sin necesidad de una consulta en el hospital o el centro de salud ${ }^{15}$. Por el lado de la oferta: la generalización de un sistema de triage para priorizar a los enfermos más graves ${ }^{16}$, los cambios de funcionamiento interno de los $\mathrm{SUH}^{17}$, el rediseño de estos servicios ${ }^{18}$, la creación de áreas específicas de observación o de corta estancia gestionadas desde urgencias ${ }^{19,20}$, un mejor equipamiento para un mayor poder resolutivo ambulatorio ${ }^{21} \mathrm{o}$ la simple adaptación a la demanda real del usuario ${ }^{22}$ han sido sólo algunas de las propuestas sugeridas y/o llevadas a cabo durante los últimos años. Parece, con todo, que la modulación de la oferta es el principal punto en el que debe incidirse. No en balde, se ha establecido de una forma clara que los determinantes internos (i.e., el propio sistema) tienen un mayor peso que los determinantes externos (i.e., la presión asistencial) en los retrasos que registran los SUH y que, entre ellos, juegan un papel fundamental la falta de camas de hospitalización para los pacientes que deben ingresar una vez que el proceso asistencial urgente ya ha terminado $y$, en menor medida, la necesidad de permanencia del paciente en el SUH de pacientes en observación pendientes de evaluar su evolución con el tratamiento administrado ${ }^{23,24}$. En todo este complejo entramado, una cosa sí parece estar clara: es necesaria una buena coordinación entre todas las estructuras asistenciales que dan respuesta a la petición de demanda de asistencia urgente ${ }^{25}$, ya que cuando aquélla se produce, sus resultados repercuten favorablemente en el usuario ${ }^{26,27}$. Y esto no implica necesariamente un incremento de los costes, ya que se ha demostrado que, en determinadas áreas, la gestión que hace de ellas un urgenciólogo resulta altamente eficiente en comparación con otros modelos más clásicos de explotación ${ }^{28}$. Así, el grupo de Salazar ha demostrado en diversas ocasiones y en distintos centros sanitarios la veracidad de esta afirmación ${ }^{29-31}$. 
Así las cosas, ¿qué nos aporta el estudio de Pérez-Ciordia y Guillén Grima que se publica en este número de Anales del Sistema Sanitario de Navarra ${ }^{32}$ Los autores realizan una descripción del usuario que demanda asistencia médica urgente hospitalaria y extrahospitaria en la Comunidad de Navarra mediante una encuesta a una muestra extensa (5.537 pacientes) y bastante representativa (el $61 \%$ del universo respecto a la cobertura hospitalaria y el $85 \%$ respecto a la extrahospitalaria) de dicha comunidad. Comprueban que la solicitud de asistencia extrahospitalaria es la más frecuente, ya que supone un $62,7 \%$ del total, y que la principal motivación para optar por esta modalidad (a la que se acogen de forma significativa los pacientes con buen estado de salud habitual y con percepción de patología leve) es la comodidad del servicio. Las patologías prototipo de este grupo serían los procesos respiratorios de vías altas, los dermatológicos y los oculares, que en más del $80 \%$ de los casos son atendidas en el entorno extrahospitalario. En cambio, la principal razón que lleva al usuario a acudir al SUH, lo cual sucede en el $37,3 \%$ de casos, es la percepción de necesidad de dicho nivel asistencial por la sensación de gravedad del proceso que le afecta. En este caso, los procesos cardiorrespiratorios y los del aparato locomotor son los ejemplos más claros, puesto que en más del 50\% de los casos su atención se hace en el SUH.

Los resultados aquí publicados están, en gran medida, en consonancia con múltiples estudios que han investigado en España la demanda de asistencia por parte del usuario y las razones que motivan ésta, tanto a nivel hospitalario como extrahospitalario ${ }^{6,33-35}$. Aunque pudiera parecer redundante el incidir de forma repetitiva en la investigación de un mismo aspecto, en este caso no lo es. Como bien es sabido, en España las competencias en materia de sanidad se encuentran transferidas a cada autonomía y, por tanto, caben variaciones locales en cuanto a la forma en la que se organiza la asistencia urgente. Ello es así porque existen diferencias en cuanto a la tipología demográfica y orográfica de cada comunidad que las justifican, y la adaptación a esta realidad da lugar a modelos o ejecuciones del modelo diferentes. Sirva como ejemplo el reciente mapa de unidades de soporte vital avanzado (USVA) presentado por Pesqueira Alonso y Juliani Izquierdo, en el que se ponía en evidencia una notable disparidad entre las comunidades autónomas en cuanto a los índices de USVA por extensión, población y densidad de población ${ }^{36}$.

Algunos datos colaterales del estudio de Pérez-Ciordia y Guillén Grima también son merecedores de algún comentario. Los extranjeros afincados en Navarra hacen una utilización proporcionalmente idéntica de las urgencias hospitalarias y extrahospitalarias que la población autóctona. En múltiples trabajos previos se ha demostrado que el patrón de utilización de estos usuarios no difiere en gran manera al resto de la población ${ }^{37-39}$. También es destacable que entre las razones individuales por las que el paciente acude al SUH y no al sistema extrahospitalario se encuentra el que haya sido remitido por el médico de cabecera (con una razón de odds (OR) de 5,5) y la mayor confianza de aquél hacia el hospital (OR: 5,1) y su mayor tecnología (OR 5,7). Finalmente, un mal estado de salud de base del paciente, que pudiera parecer determinante de un mayor uso de los SUH, no lo es si se ajusta 
por la edad y el sexo de los pacientes. De todo lo dicho parece, pues, desprenderse que el usuario no ejerce sus derechos de elegir uno u otro tipo de asistencia urgente con tanta arbitrariedad o espontaneidad como en ocasiones se piensa.

El estudio también tiene algunas limitaciones que conviene tener presentes. Una limitación importante es que los autores consiguieron un porcentaje de respuestas bajo, por debajo del $50 \%$ de las encuestas distribuidas, y ello genera incertidumbre acerca de un posible sesgo a la hora de extrapolar los resultados de las encuestas recibidas a toda la población. Por otro lado, su muestra no incluyó las consultas hospitalarias y extrahospitalarias más graves (niveles I y II en un sistema de triage de 5 niveles), las urgencias hospitalarias obstétrico-ginecológicas, las atendidas en el domicilio ni los accidentes de tráfico. Aunque a buen seguro existieron razones de peso que hicieron que se excluyeran estos pacientes en el diseño de un estudio tan ambicioso como éste, debe reconocerse que si el modelo hubiese perseguido una muestra procedente de un universo sin excepciones podría haber acercado más si cabe los resultados a la realidad navarra.

A la postre, la realidad actual en España es que los usuarios saben que tienen a su libre disposición toda una estructura que vela de forma permanente, en sus múltiples formas, por el mantenimiento de su salud en casos de urgencia. Siempre está allí, cercana y resolutiva en el problema agudo. Sirva de ejemplo la actividad desarrollada y la atención prestada durante los primeros días del brote de gripe A $(\mathrm{H} 1 \mathrm{~N} 1)^{40,41}$. Sin embargo, el engranaje de esta estructura no está suficientemente lubricado. Y, en este escenario, creo que es el momento de considerar de una vez por todas que lo que es erróneo no son las expectativas del usuario, pues probablemente no sean corregibles, ni siquiera moldeables, en el corto, medio o largo plazo, sino la respuesta que el sistema da a ellas. Comparto con Moreno Millán ${ }^{42}$ el punto de vista que el problema del desequilibrio entre demanda y oferta de atención médica urgente ya no se trata de un problema, sino de una transformación en la cultura social a la que las soluciones propuestas hasta la fecha no han dado respuesta adecuada, y que debe ser asumida cuanto antes como tal para poder implantar las herramientas y mecanismos necesarios que permitan poner fin a una situación de desencuentro que, más que solucionarse, se ha enquistado con los años.

\section{BIBLIOGRAFÍA}

1. SAN LuCAS. Parábola del buen samaritano. El Nuevo Testamento: Evangelio según San Lucas. Capítulo 10, vers. 25 al 37.

2. Ministerio de Sanidad y Política Social. Estadística de hospitales: estadística de establecimientos con régimen de internado. (Accedido el 3 de octubre de 2009). Disponible en: www.msc.es/estadEstudios/estadisticas/sisInfSanSNS/ actDesarrollada.htm

3. SÁnchez M. ¿Urgencias inadecuadas u oferta insuficiente? Med Clin (Barc) 2004; 123: 619-620.

4. Salgado E, Antolín A, Rodríguez D, Bragulat E, Sánchez M, Miró O. Cuantificación de los efectos negativos de la sobrecarga invernal en urgencias y de la efectividad de las medidas extraordinarias invernales para paliarlos. Med Clin (Barc) 2008; 130: 286-291. 
5. Sánchez M, Miró O, Coll-Vinent B, Bragulat E, Espinosa G, Gómez-Angelats E et al. Saturación del servicio de urgencias: factores asociados y cuantificación. Med Clin (Barc) 2003; 121: 167-172.

6. Aranaz Andrés JM, Martínez Nogueras R, Rodrigo Bartual V, Gómez Pajares F, ANTÓN GARCíA P. Adecuación de la demanda de atención sanitaria en servicios de urgencias hospitalarios. Med Clin (Barc) 2004; 123: 615-618.

7. Márquez Cabeza JJ, Domínguez Quintero B, Méndez Sánchez J, Gómez Álvarez B, Toronjo Romero S, Del Río Morales E et al. Diferencias en los motivos de consulta entre pacientes que acuden derivados y por iniciativa propia a un Servicio de Cuidados Críticos y Urgencias de un hospital general. Emergencias 2007; 19: 70-76.

8. SÁnchez M, Smally AJ. Comportamiento de un servicio de urgencias según el día de la semana y el número de visitas. Emergencias 2007; 19: 319-22.

9. Miró O, SÁnchez M, Borrás A, Millá J. Fútbol, televisión y servicios de urgencias. Med Clin (Barc) 2000; 114: 538-539.

10. Instituto Nacional de Estadística. Padrón de habitantes. (Accedido el 2 de octubre de 2009). Disponible en: www.ine.es

11. Moreno Millán E, García Torrecillas JM, Lea Pereira MC. Diferencias de gestión entre los ingresos urgentes y los programados en función de los grupos relacionados de diagnóstico y la edad de los pacientes. Emergencias 2007; 19: 122-128.

12. Miró O, SÁnchez M, Coll-Vinent B, Millá J. Indicadores de calidad en urgencias: comportamiento en relación con la presión asistencial. Med Clin (Barc) 2001; 116: 92-97.

13. Moreno Millán E. Ventajas e inconvenientes del copago en la financiación y gestión de la atención sanitaria urgente. Emergencias 2007; 19: 32-35.

14. Miró O, Salgado E, Tomás S, Espinosa G, Estrada C, Martí C et al. Derivación sin vista desde los servicios de urgencias hospitalarios: cuantificación, riesgos y grado de satisfacción. Med Clin (Barc) 2006; 126: 88-93.

15. Coma E, De la Haba I, Comabella R, Zayas S, Armisen A, Ciurana R et al. La atención telefónica urgente especializada: una manera de mejora en la utilización de los servicios de urgencias hospitalarios. Emergencias 2009; 21: 117-120.

16. Martín-SÁnchez FJ, GonzÁlez-Del Castillo J, Zamorano J, Candel FJ, González-ArmenGOL JJ, VILLARROEL P et al. El facultativo, un elemento necesario en el triaje de un Servicio de Urgencias en un hospital terciario. Emergencias 2008; 20: 41-47.

17. Sánchez M, Salgado E, Miró O. Mecanismos organizativos de adaptación y supervivencia de los servicios de urgencia. Emergencias 2008; 20: 48-53.

18. Miró O, Sánchez M, Espinosa G, Coll-Vinent B, Bragulat E, Milla J. Analysis of patient flow in the emergency department and the effect of an extensive reorganisation. Emerg Med J 2003; 20: 143-148.

19. Aldea-Molina E, Gómez J, Royo R, Rodrigo G, Rivas M, Llera R. Sala de observación de un servicio de urgencias: un lugar adecuado para el manejo del flutter auricular. Emergencias 2008; 20: 101-107.

20. González-Armengol JJ, Fernández Alonso C, Martín-SÁnchez FJ, GonzÁlez-Del CastiLLO J, LóPEz-FARRÉ A, Elvira C et al. Actividad de una unidad de corta estancia en urgencias de un hospital terciario: cuatro años de experiencia. Emergencias 2009; 21 : 87-94.

21. Anónimo. Las urgencias hospitalarias, a la baja en el ICS. Diario Médico, 31/07/2008, p4.

22. Moreno Millán E. ¿Y si adaptáramos los servicios hospitalarios de urgencias a la demanda social y no a las necesidades de salud? Emergencias 2008; 20: 276-284.

23. Espinosa G, Miró O, Coll-Vinent B, SÁnchez M, Millá J. Effects of internal and external factors on emergency department overcrowding. Ann Emerg Med 2002; 39: 693-695. 
24. Miró O, SÁncheZ M, Coll-Vinent B, Millá J. Estimación del efecto relativo que ejercen los determinantes externos e internos sobre la eficacia de un servicio de urgencias de medicina. Med Clin (Barc) 2000; 115: 294-296.

25. Montero Pérez FJ. La organización de la cadena asistencial urgente en España o la búsqueda de los eslabones perdidos. Emergencias 2007; 20: 5-7.

26. García Bermejo P, Mínguez Platero J, Ruiz López JL, Millán Soria J, Trescoli Serrano C, TARAZONA Ginés E. Gestión integral del área de urgencias y coordinación con atención primaria: Emergencias 2008; 20: 8-14.

27. Jiménez S, De la Red G, Miró O, Bragulat E, Coll-Vinent B, Senar E et al. Efectividad de la incorporación de un médico especialista en medicina familiar y comunitaria en un servicio de urgencias hospitalario. Med Clin (Barc) 2005; 125: 132-137.

28. SalaZAR A. ¿Urgenciólogos rentables? Emergencias 2009; 21: 83-84.

29. Juan A, Salazar A, Álvarez A, Pérez JR, García L, Corbella X. Effectiveness and safety of an emergency department short-stay unit as an alternative to standard inpatient hospitalisation. Emerg Med J 2006; 23: 833-837.

30. Salazar A, Juan A, Ballbe R, Corbella X. Emergency short-stay unit as an effective alternative to in-hospital admission for acute chronic obstructive pulmonary disease exacerbation. Am J Emerg Med 2007; 25: 486-487.

31. Salazar A, Estrada C, Porta R, Lolo M, Tomás S, Álvarez M. Home hospitalization unit: an alternative to standard inpatient hospitalization from the emergency department. Eur J Emerg Med. 2009; 16: 121-123.

32. Pérez-Ciordia I, Guillén Grima F. Urgencias hospitalarias y extrahospitalarias en Navarra. Razones que las motivan. An Sist Sanit Navar 2009; 32: 371-384.

33. Sempere T, Peiró S, Sendra P, Martínez C, López I. Inappropriate use of an accident and emergency department: magnitude, associated factors and reasons- an approach with explicit criteria. Ann Emerg Med 2001; 37: 568-579.

34. Ortega Maján MT, Rabanaque Hernández MJ, Júdez Legaristi D, Cano Del Pozo Mi, Abad Dízz JM, Moliner Lahoz J. Perfil de los usuarios y motivos de demanda del Servicio de Urgencias extrahospitalario 061. Emergencias 2008; 20: 27-34.

35. Cano del Pozo Mi, Rabanaque Hernández MJ, Feja Solana C, Martos Jiménez MC, Abad Díez JM, Celorrio Pascual JM. Estudio de la frecuentación de un servicio de urgencias extrahospitalario. Emergencias 2008; 20: 179-186.

36. Pesqueira Alonso EE, Juliani Izquierdo P. Unidades de soporte vital avanzado en España 2008. Mapa de situación. Emergencias 2009; 21: 276-282.

37. Cots F, Castells X, García O, Riu M, Felipe A, Vall O. Impact of immigration on the cost of emergency visits in Barcelona (Spain). BMC Health Serv Res 2007; $7: 9$.

38. JunYent M, NúÑEz S, Miró O. Medical emergencies in the adult immigrant. An Sist Sanit Navar 2006; 29 (Supl 1): 27-34.

39. JunYent M, Miró O, SÁnchez M. Comparación de la utilización de los servicios de urgencias hospitalarios entre la población inmigrante y la población autóctona. Emergencias 2006; 18: 232-235.

40. Miró O, Rovira E, Blanco AJ, Salmerón JM, Gatell JM, Cervera C et al. Descripción clínica y epidemiológica de los primeros casos de gripe nueva A (H1N1) atendidos en España. Emergencias 2009; 21: 166-171.

41. González del Castllo J. La gripe nueva A (H1N1): papel de los servicios de urgencias como atalaya y primera línea de choque frente a brotes epidémicos. Emergencias 2009; 21: 162-163.

42. Moreno Millán E. Servicios de urgencias y listas de espera. Emergencias 2007; 19: 57-58. 